\title{
Chitosan Modulates Inflammatory Responses in Rats Infected with Enterotoxigenic Escherichia coli
}

\author{
Gang Liu, ${ }^{1,2}$ Shuai Chen, ${ }^{2}$ Guiping Guan, ${ }^{1}$ Jun Tan, ${ }^{1}$ Naif A. Al-Dhabi, ${ }^{3}$ Hongbing Wang, \\ Veeramuthu Duraipandiyan, ${ }^{3}$ and Jun Fang ${ }^{1}$ \\ ${ }^{1}$ College of Bioscience and Biotechnology, Hunan Agricultural University, Changsha, Hunan 410128, China \\ ${ }^{2}$ Institute of Subtropical Agriculture, Chinese Academy of Sciences, Scientific Observing and Experimental Station of \\ Animal Nutrition and Feed Science in South-Central, Ministry of Agriculture, Hunan Provincial Engineering Research Center of \\ Healthy Livestock, Key Laboratory of Agro-Ecological Processes in Subtropical Region, Changsha, Hunan 410125, China \\ ${ }^{3}$ Department of Botany and Microbiology, Addiriyah Chair for Environmental Studies, College of Science, King Saud University, \\ P.O. Box 2455, Riyadh 11451, Saudi Arabia \\ ${ }^{4}$ Hunan Institute of Animal and Veterinary Science, Changsha, Hunan 410131, China
}

Correspondence should be addressed to Guiping Guan; guanguiping@hunau.edu.cn and Hongbing Wang; hongbingwanggg@gmail.com

Received 18 September 2016; Revised 16 November 2016; Accepted 30 November 2016

Academic Editor: Michael Conlon

Copyright (C) 2016 Gang Liu et al. This is an open access article distributed under the Creative Commons Attribution License, which permits unrestricted use, distribution, and reproduction in any medium, provided the original work is properly cited.

\begin{abstract}
This study aims to investigate the effects of dietary chitosan (COS) on gastrointestinal pathogen resistance in mice model. For two weeks, a control group of ICR mice received a basal diet whilst the intervention group received the basal diet supplemented with $300 \mathrm{mg} / \mathrm{kg}$ COS. After two weeks, the mice fed the supplemented diet had a lower body weight. Then enterotoxigenic Escherichia coli (E. coli) was administered to the mice through oral gavage, with each mouse receiving $10^{8} \mathrm{CFU}$. At day 7 after infection, the bacterial load in the jejunum and faeces was significantly lower in the COS group than that in the control group. Moreover, the mRNA and protein levels of IL-1 $\beta$, IL-6, IL-17, IL-18, and TNF- $\alpha$ were significantly lower in the group of mice receiving the COS diet; also the jejunal production of toll-like receptor-4 (TLR-4) was suppressed in the COS group. These results indicate the intervention influenced inflammation and controlled E. coli infection.
\end{abstract}

\section{Introduction}

For most people, enterotoxigenic Escherichia coli (ETEC) infection is merely the unpleasant and inconvenient cause of traveller's diarrhoea in many instances. ETEC is responsible for the majority of diarrhoeal disease in developing countries; according to WHO it claims approximately 380,000 lives each year [1] with children under the age of 5 years being especially vulnerable. ETEC is also prevalent amongst newborn farm animals, such as piglets [2].

ETEC infections usually emerge 1-3 days following pathogen exposure and manifest as acute watery, diarrhoea together with fever, headache, and vomiting. These symptoms typically last for 3-4 days though some people may experience diarrhoea for a week or longer [3]. ETEC colonizes the small intestine and releases enterotoxins that cause intestinal epithelial cells (IEC) to secrete fluids into the gut lumen, resulting in diarrhoea [4]. To minimise the effects of ETEC in weaning piglets, antibiotics are frequently added to their diet, with the intention of minimising infectious disease and promoting growth. However, this practice is likely to contribute to drug-resistance in pathogens by creating a reservoir of drug-resistant bacteria. These bacteria may transfer resistance genes to other pathogenic bacteria in the gut of animals and humans [5]. Also, the presence of drug residues is a concern for many consumers; therefore, the routine antibiotics in livestock diets is already banned or restricted in many countries.

Chitosan is an abundant nitrogenated polysaccharide; it is a component of fungal cell walls and exoskeletons of insects and diverse sea creatures, such as crustaceans, squid, and clams. Chitosan is derived from chitin. The immune 
responses of pigs [6], mice [7], rats [8], and fish [9] have been modulated by chitosan. T cells and other immune cells produce cytokines, which are essential to the immune response. In vitro studies indicate that the degree of deacetylation and molecular weight of COS are positively correlated with the extent of the anti-inflammatory effect [10]. Whilst some studies have endeavoured to evaluate the effects of chitosan on the gut bacteria of pigs and chickens [11] the effects of chitosan on inflammatory and bacterial responses during and after infection remain limited. One possible mechanism by which chitosan may operate is to disrupt E. coli adhesion in the jejunum.

The hypothesis that underlies this study is that dietary chitosan supplements may directly clear enterotoxigenic $E$. coli and reduce proinflammatory signals and/or increase the anti-inflammatory response during infection. We tested the hypothesis in enterotoxigenic E. coli infection model; the changes in the bacterial count and proinflammatory molecules were evaluated.

\section{Mice and Diet}

This experiment used 20 ICR (Institute for Cancer Research) mice aged 6 weeks; they were bred and kept at Hunan Agricultural University. Approval for all experimental procedures with the mice was granted by the Animal Care and Use Committees of Hunan Agricultural University. The mice were housed separately in pathogen-free accommodation under appropriate environmental conditions $\left(25^{\circ} \mathrm{C}\right.$; $53 \%$ relative humidity; 12-hour light/dark cycle). They had ad lib access to an appropriate rodent diet [12] and water. Mice were randomly allocated to two groups (control $n=10$ and chitosan intervention $n=10)$. Endotoxin free chitosan (average molecular weight $<1 \mathrm{kDa}$; degree of deacetylation $>$ 95\%) was donated by Dalian Chemical and Physical Institute (Chinese Academy of Sciences, Dalian, China). For two weeks, the control group received the basal diet and the intervention group was given a basal diet supplemented with $300 \mathrm{mg} / \mathrm{kg}$ of chitosan. The dosage and duration were determined based upon previous study [6]. Throughout the experimental period, the intake of feed and water, together with body weight gain, was monitored and recorded.

\section{Enterotoxigenic E. coli Infection and Enumeration}

After two weeks of being on a basal or supplemented diet, mice were then infected with $10^{8} \mathrm{CFU}$ ETEC SEC470 [13]. At 7 day after infection, all the mice were killed by $\mathrm{CO}_{2}$ asphyxiation. Jejunal tissues were homogenised, serially diluted, and then plated onto MacConkey agar with the antibiotic gentamicin $(40 \mu \mathrm{g} / \mathrm{mL})$ and tetracycline $(50 \mu \mathrm{g} / \mathrm{mL})$. The jejunal contents and faeces were weighted and then suspended in PBS buffer. Then serial dilutions were placed on MacConkey agar treated with gentamicin $(40 \mu \mathrm{g} / \mathrm{mL})$ and tetracycline $(50 \mu \mathrm{g} / \mathrm{mL})$. After 24 hours, the bacterial colonies were tallied. PCR was conducted to verify the identity of the bacteria isolated. The primers used ( $5^{\prime}$-CTGTATACGTGGCAG$\left.3^{\prime}\right)$ and $\left(5^{\prime}\right.$-ACTATGGTGAATGCTCAC- $\left.3^{\prime}\right)$ were obtained from ETEC fedF gene (GenBank accession number Z26520). The other jejunal tissues, jejunal contents, and faecal samples were collected and stored at $-80^{\circ} \mathrm{C}$ until required.

\section{RT-PCR and ELISA Analysis of Cytokines}

After mRNA was extracted using TRIZOL reagent (Invitrogen, USA), cDNA was reverse transcribed. RT-PCR was performed according to Xiao et al. [14]. The mRNA levels of $I L-1 \beta, I L-6, I L-17, I L-18$, and TNF- $\alpha$ were analysed with GAPDH as the reference gene. The protein levels of $I L-1 \beta, I L-$ $6, I L-17, I L-18$, and $T N F-\alpha$ were analysed according to Ren et al. [15] with ELISA kit from eBioscience, CA, USA.

\section{Immunoblotting of TLR-4}

To measure the level of TLR-4, an appropriate TLR-4 assay kit was used (Cayman Chemical Company, MI, USA). Equal quantities of proteins collected from jejunal tissues were separated using SDS-PAGE. Then the samples were analysed according to the method described by a previous report [16]. Using $\beta$-actin protein as a reference, the intensity of the signal was measured digitally.

\section{Statistical Analyses}

The data are presented as means \pm standard error of the mean (SEM). All the statistical analyses were undertaken using SPSS 22.0 software (Chicago, IL, USA). The Student's $t$-test was used to analyse data differences between the control and intervention groups. Difference level at $P<0.05$ is considered significant.

\section{Results}

Over the two-week period, the average feed and water intake was higher in the in chitosan-supplemented mice than in control mice $(P<0.05)$ (Figures $1(\mathrm{a})$ and $1(\mathrm{~b}))$. Despite this, the body weight of mice receiving the chitosan supplement was significantly lower than that of control group $(P<$ 0.05) (Figure 1(c)). During the experiment, no diarrhoea was observed. At 7 days after infection, in the COS group, the ETEC loads in the jejunal contents, faeces, and jejunal tissues were significantly lower $(P<0.05)$ than that in the control group (Figure 2).

Seven days after infection, the expression of $I L-1 \beta, I L$ 6, $I L-17, I L-18$, and TNF- $\alpha$ mRNA was significantly lower in the jejunum of COS-fed mice compared to those of controls (Figure 3 ). The ELISA results confirmed the trend with IL- $1 \beta$, IL-6, IL-17, IL-18, and TNF- $\alpha$ being significantly lower in COS mice (Table 1).

As Figure 4 indicates in the COS group the TLR4 protein level in the jejunum was significantly lower $(P<0.05)$ than that of the control group.

\section{Discussion}

Numerous studies have suggested that dietary supplements of chitosan can reduce body weight and may have applications 


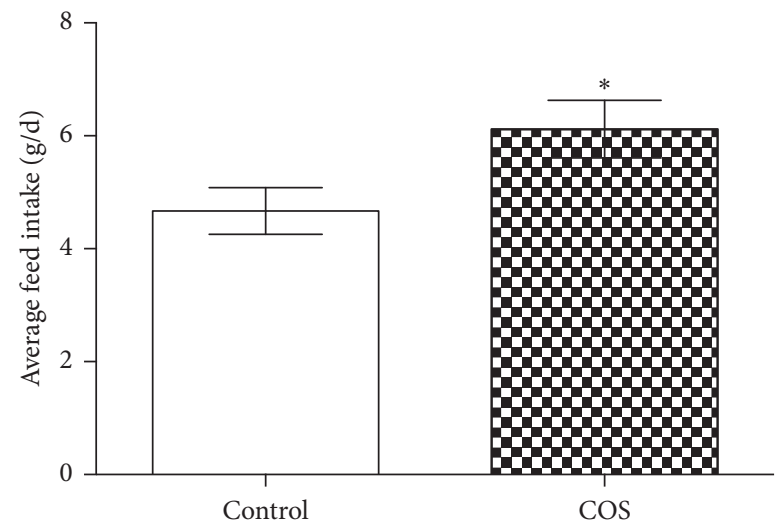

(a)

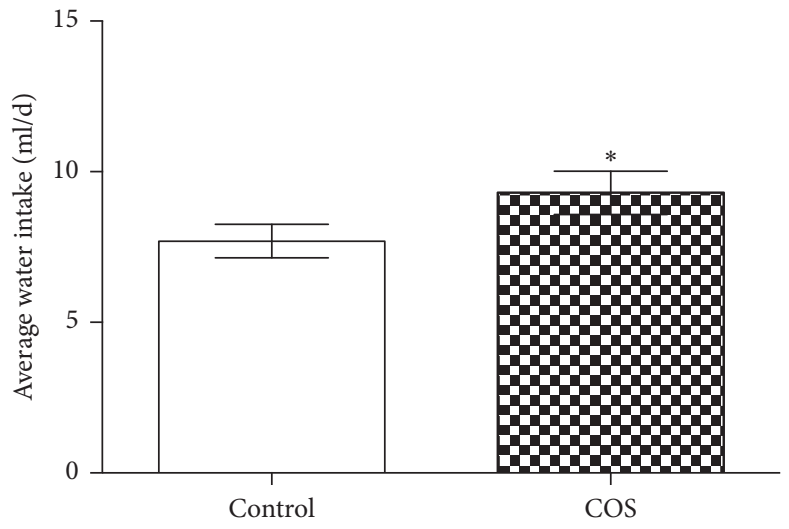

(b)

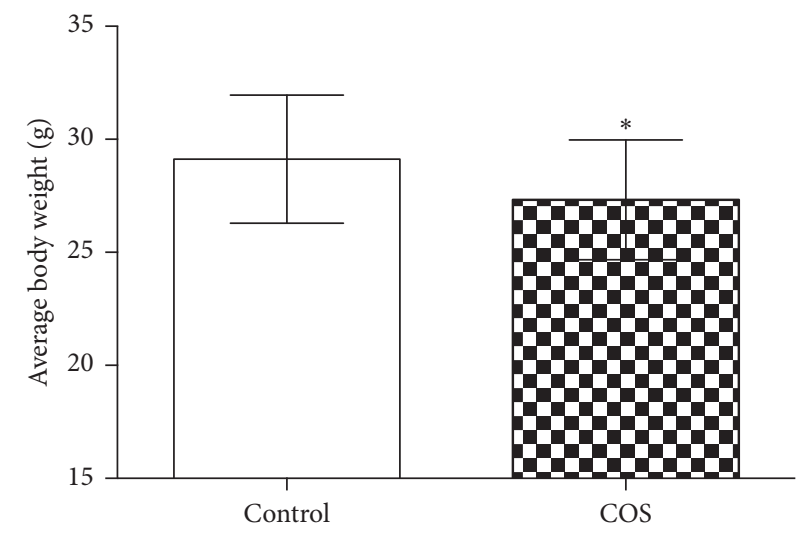

(c)

Figure 1: Chitosan supplementation lowers mouse body weight. (a) Average feed intake in the control group and COS group. (b) Average water intake in the control group and COS group. (c) Average body weight in the control group and COS group. * indicates a significant difference between the control and COS groups $(P<0.05)(n=10)$.

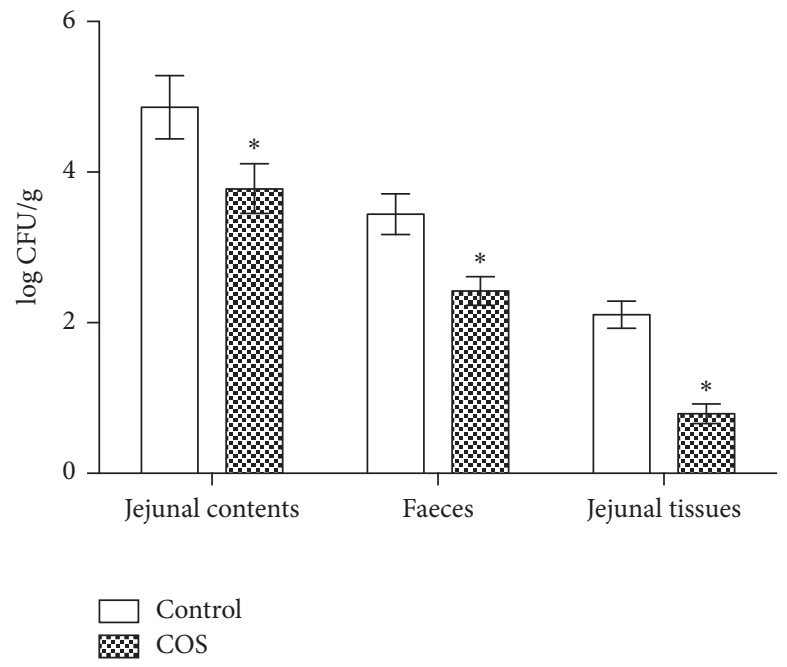

Figure 2: ETEC load at $\mathrm{d} 7$ after infection from jejunal tissues, jejunal contents, and faeces of infected ICR mice $(n=10) . *$ indicates a significant difference between the control and COS groups $(P<0.05)$. 


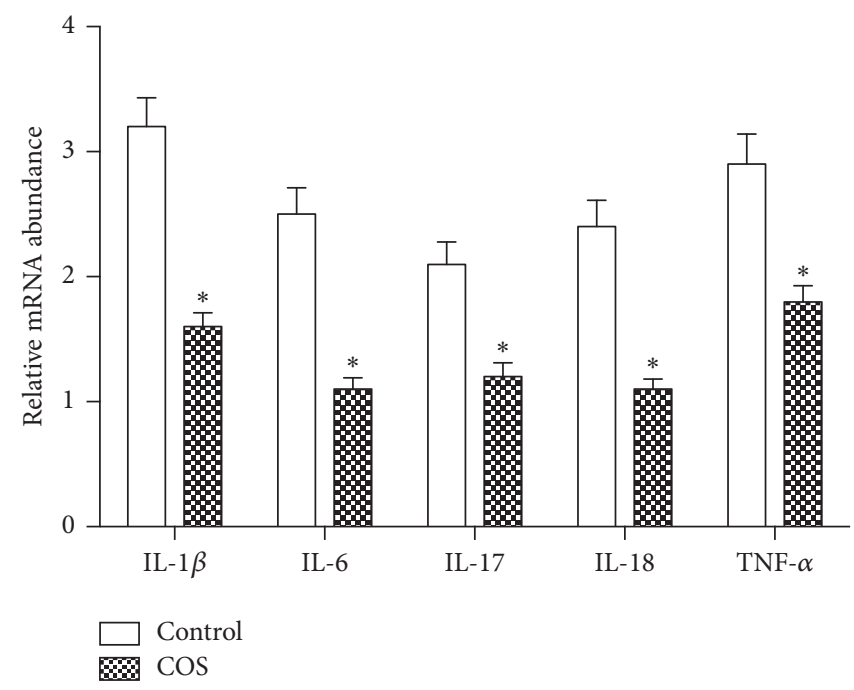

FIgURE 3: Mucosal inflammatory responses in the jejunum from the control group and COS group $(n=6)$; mRNA level of $I L-1 \beta, I L-6, I L-17$, $I L-18$, and TNF- $\alpha$ as determined by RT-PCR. $*$ indicates a significant difference between the control and COS groups $(P<0.05)$.

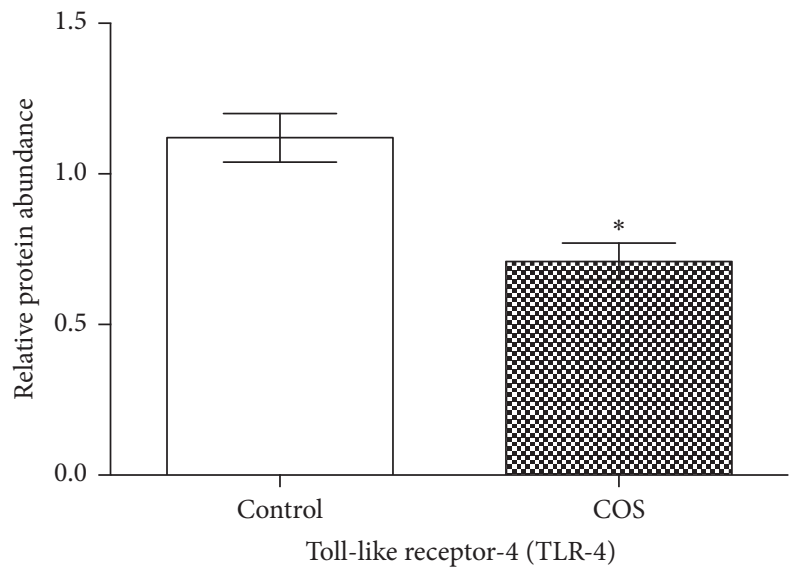

FIGURE 4: TLR4 protein level in jejunal mucosa in the control group and COS group $(n=6) . *$ indicates a significant difference between the control and COS groups $(P<0.05)$.

for weight management and obesity $[7,17]$. During the experimental period, the mice receiving $10^{8}$ CFU ETEC SEC470 did not suffer any diarrhoea. Many researches showed that the mice challenged by ETEC would not suffer from diarrhoea; however, ETEC could colonize the intestine and promote the inflammatory responses [18]. Thus we further investigated the body weight of the mice. The results demonstrate that mice fed a chitosan-supplemented diet experienced a reduction in body weight. One mechanism that has been proposed for this is that chitosan reduces the postprandial ratios of apolipoprotein B (apoB) isoforms, low-density lipoprotein cholesterol, and high-density lipoprotein. This is based on the findings of a study of the effects on lipid metabolism in glucose-tolerant rats receiving a high-sucrose diet [19]. The chitosan particles bind with cholesterol and fatty acids to form clusters in the gastrointestinal tract, thereby reducing lipid absorption [20]. In addition to its effect on lipid absorption, chitosan has been described as reducing blood glucose levels and being capable of inhibiting the carbohydrate hydrolysing enzymes maltase, sucrose, and sucrose-isomaltase in the gut [21,22].

The findings from this study revealed that, compared to the control group, the COS mice had a significantly lower load of ETEC bacteria. According to the report of Tayel, chitosan may inhibit the growth of bacteria such as $E$. coli, Enterobacteriaceae, and Staphylococcus aureus [23]. As $\mathrm{COS}$ is a $\mathrm{D}$-glucosamine oligomer, it is resistant to digestive enzyme degradation. COS may survive digestive enzymes to reach the jejunum where it interferes with the adhesion of ETEC to intestinal epithelial cells.

In this study the mRNA and protein levels of jejunal IL$1 \beta$, IL- 6 , TNF- $\alpha$, and TLR- 4 were observed in response to a chitosan-supplemented diet in mice. Intestinal epithelial cells express cytokine and chemokine receptors as well as tolllike receptors, such as TLR-4 [16]. TLR4 recruits the adaptor protein, MyD88, and TIRAP to activate NF- $\kappa \mathrm{B}$, which in turn induces expression of signalling genes such as TNF- $\alpha$, IL-1, 
TABLE 1: Jejunal inflammatory responses in jejunum tissues from the control group and COS group $(n=6)$ and protein levels of IL-1 $\beta$, IL-6, IL-17, IL-18, and TNF- $\alpha$ were determined by ELISA.

\begin{tabular}{lcr}
\hline & Control & COS \\
\hline IL-1 $\beta(\mathrm{pg} / \mathrm{ml})$ & $110.48 \pm 8.31$ & $67.59 \pm 4.67^{*}$ \\
IL-6 $(\mathrm{pg} / \mathrm{ml})$ & $98.45 \pm 6.38$ & $42.36 \pm 3.58^{*}$ \\
IL-17 $(\mathrm{pg} / \mathrm{ml})$ & $216.39 \pm 11.23$ & $117.96 \pm 9.67^{*}$ \\
IL-18 $(\mathrm{pg} / \mathrm{ml})$ & $182.64 \pm 13.62$ & $98.63 \pm 8.45^{*}$ \\
TNF- $\alpha(\mathrm{pg} / \mathrm{ml})$ & $136.25 \pm 7.12$ & $86.23 \pm 8.31^{*}$ \\
\hline
\end{tabular}

* indicates a significant difference between the control and COS groups $(P<0.05)$.

and IL-6 [18]. Raised TLR-4 expression in intestinal epithelial cells is associated with an enhanced mucosal inflammatory response and subsequent dysfunction of the intestinal epithelial barrier [18]. Our study revealed suppressed expression of TLR-4 and consequently the mRNA and protein levels of IL-1 $\beta$, IL- 6 , and TNF- $\alpha$ were correspondingly lower. The anti-inflammatory effects of COS on mice are proposed to arise from the COS inhibition of intestinal ETEC infection. It is interesting in our previous research, the mRNA levels of IL- $1 \beta$, and IL- 6 in the ETEC-challenged piglets consuming $300 \mathrm{mg} / \mathrm{kg}$ COS diet were significantly higher than those in the control group [6]. This discrepancy may be due to two reasons: firstly, it may take more times for complex network of cytokines in piglets to respond to the COS diet; thus the IL- $1 \beta$ and IL- 6 mRNA levels in jejunal mucosa were still high during the sampling; secondly, the samples used for mRNA analysis are different, the jejunal mucosa was used for analysis in piglets model and the jejunal tissues were used in the mice model.

The findings here also indicated that the jejunal expression of IL-17 and IL-18 was reduced by the chitosan supplement. IL-17, manufactured in response to inducible (iTh17) and natural (nTh17) T helper cells, is a proinflammatory cytokine that recruits monocytes and neutrophils to areas of inflammation; it also stimulates local chemokine synthesis. It has been implicated in a number of autoimmune diseases [24]. On the other hand, IL-18, another proinflammatory cytokine, is strongly implicated in antitumour responses and host defence. Gene therapy studies that increase of IL-18 has been shown to provide test animals with protection against infection, tumour growth, and metastases. However, overexpression of IL-18 has been shown to result in emphysematous lesions in mice [25]. Chitosan lowered the bacterial load in jejunum, and the lower ETEC load may decrease intestinal expression of cytokines.

To summarise, chitosan supplements decrease the body weight of mice and are an effective prophylactic against in vivo infection of enterotoxigenic E. coli. The results presented here indicate that COS decreased the bacterial load, TLR-4, and cytokine biosynthesis. Further development of COS may provide an effective method to promote intestinal health and protection against enterotoxigenic E. coli infection.

\section{Competing Interests}

The authors declare that there is no conflict of interests regarding the publication of this article.

\section{Acknowledgments}

This project was supported by International Partnership Program of Chinese Academy of Sciences (161343KYSB20160008), National Key Research and Development Program of China (2016YFD0500504), National Natural Science Foundation of China (nos. 31672457 and 31402092), Ministry of Agricultural of the People's Republic of China (2015-Z64 and 2016-X47), and Hunan Provincial Science and Technology Department (2016NK2101, 2016WK2008, and 2016TP2005). This project was also partially financially supported by King Saud University, through Vice Deanship of Research Chairs.

\section{References}

[1] "Future directions for research on enterotoxigenic Escherichia coli vaccines for developing countries," The Weekly Epidemiological Record, vol. 81, no. 11, pp. 97-104, 2006.

[2] J. M. Fairbrother, E. Nadeau, and C. L. Gyles, "Escherichia coli in postweaning diarrhea in pigs: an update on bacterial types, pathogenesis, and prevention strategies," Animal Health Research Reviews, vol. 6, no. 1, pp. 17-39, 2005.

[3] J. S. Yoder, S. Cesario, V. Plotkin, X. Ma, K. Kelly-Shannon, and M. S. Dworkin, "Outbreak of enterotoxigenic Escherichia coli infection with an unusually long duration of illness," Clinical Infectious Diseases, vol. 42, no. 11, pp. 1513-1517, 2006.

[4] P. Liu, X. S. Piao, P. A. Thacker et al., "Chito-oligosaccharide reduces diarrhea incidence and attenuates the immune response of weaned pigs challenged with Escherichia coli K88," Journal of Animal Science, vol. 88, no. 12, pp. 3871-3879, 2010.

[5] H. J. van der Fels-Klerx, L. F. Puister-Jansen, E. D. van Asselt, and S. L. G. E. Burgers, "Farm factors associated with the use of antibiotics in pig production," Journal of Animal Science, vol. 89, no. 6, pp. 1922-1929, 2011.

[6] D. Xiao, Y. Wang, G. Liu et al., "Effects of chitosan on intestinal inflammation in weaned pigs challenged by enterotoxigenic Escherichia coli," PLoS ONE, vol. 9, no. 8, Article ID e104192, 2014.

[7] E. H. Choi, H. P. Yang, and H. S. Chun, "Chitooligosaccharide ameliorates diet-induced obesity in mice and affects adipose gene expression involved in adipogenesis and inflammation," Nutrition Research, vol. 32, no. 3, pp. 218-228, 2012.

[8] I.-M. Fang, C.-M. Yang, and C.-H. Yang, "Chitosan oligosaccharides prevented retinal ischemia and reperfusion injury via reduced oxidative stress and inflammation in rats," Experimental Eye Research, vol. 130, pp. 38-50, 2015.

[9] C. Qin, Y. Zhang, W. Liu, L. Xu, Y. Yang, and Z. Zhou, "Effects of chito-oligosaccharides supplementation on growth 
performance, intestinal cytokine expression, autochthonous gut bacteria and disease resistance in hybrid tilapia Oreochromis niloticus $\$ \times$ Oreochromis aureus đơ," Fish \& Shellfish Immunology, vol. 40, no. 1, pp. 267-274, 2014.

[10] S.-H. Lee, M. Senevirathne, C.-B. Ahn, S.-K. Kim, and J.-Y. Je, "Factors affecting anti-inflammatory effect of chitooligosaccharides in lipopolysaccharides-induced RAW264.7 macrophage cells," Bioorganic \& Medicinal Chemistry Letters, vol. 19, no. 23, pp. 6655-6658, 2009.

[11] C. M. Yang, P. R. Ferket, Q. H. Hong et al., "Effect of chitooligosaccharide on growth performance, intestinal barrier function, intestinal morphology and cecal microflora in weaned pigs," Journal of Animal Science, vol. 90, no. 8, pp. 2671-2676, 2012.

[12] W. Ren, S. Chen, J. Yin et al., "Dietary arginine supplementation of mice alters the microbial population and activates intestinal innate immunity," Journal of Nutrition, vol. 144, no. 6, pp. 988995, 2014.

[13] H. Liu, L. Song, Y. Cai, Y. Wang, and L. Yu, "Draft genome sequence of Escherichia coli strain SEC470, isolated from a piglet experiencing diarrhea," Genome Announcements, vol. 4, no. 2, pp. e00088-16, 2016.

[14] D. Xiao, W. Ren, P. Bin et al., "Chitosan lowers body weight through intestinal microbiota and reduces IL-17 expression via mTOR signalling," Journal of Functional Foods, vol. 22, pp. 166176, 2016.

[15] W. Ren, S. Chen, L. Zhang et al., "Interferon tau affects mouse intestinal microbiota and expression of IL-17," Mediators of Inflammation, vol. 2016, Article ID 2839232, 9 pages, 2016.

[16] M. T. Abreu, E. T. Arnold, L. S. Thomas et al., "TLR4 and MD-2 expression is regulated by immune-mediated signals in human intestinal epithelial cells," Journal of Biological Chemistry, vol. 277, no. 23, pp. 20431-20437, 2002.

[17] A. M. Neyrinck, L. B. Bindels, F. De Backer, B. D. Pachikian, P. D. Cani, and N. M. Delzenne, "Dietary supplementation with chitosan derived from mushrooms changes adipocytokine profile in diet-induced obese mice, a phenomenon linked to its lipid-lowering action," International Immunopharmacology, vol. 9, no. 6, pp. 767-773, 2009.

[18] J. Tourret, B. P. Willing, M. A. Croxen et al., "Small intestine early innate immunity response during intestinal colonization by Escherichia coli depends on its extra-intestinal virulence status," PLOS ONE, vol. 11, no. 4, Article ID e0153034, 2016.

[19] S. Liu, S. He, and M. Chiang, "Effects of long-term feeding of chitosan on postprandial lipid responses and lipid metabolism in a high-sucrose-diet-impaired glucose-tolerant rat model," Journal of Agricultural and Food Chemistry, vol. 60, no. 17, pp. 4306-4313, 2012.

[20] R. A. A. Muzzarelli, F. Orlandini, D. Pacetti et al., "Chitosan taurocholate capacity to bind lipids and to undergo enzymatic hydrolysis: an in vitro model," Carbohydrate Polymers, vol. 66, no. 3, pp. 363-371, 2006.

[21] S.-H. Jo, K.-S. Ha, K.-S. Moon et al., "Molecular weight dependent glucose lowering effect of low molecular weight chitosan oligosaccharide (GO2KA1) on postprandial blood glucose level in SD rats model," International Journal of Molecular Sciences, vol. 14, no. 7, pp. 14214-14224, 2013.

[22] J.-G. Kim, S.-H. Jo, K.-S. Ha et al., "Effect of long-term supplementation of low molecular weight chitosan oligosaccharide (GO2KA1) on fasting blood glucose and HbAlc in $d b / d b$ mice model and elucidation of mechanism of action," $B M C$
Complementary and Alternative Medicine, vol. 14, no. 1, article 272, 2014.

[23] A. A. Tayel, "Microbial chitosan as a biopreservative for fish sausages," International Journal of Biological Macromolecules, vol. 93, pp. 41-46, 2016.

[24] W. Ouyang, J. K. Kolls, and Y. Zheng, "The biological functions of T helper 17 cell effector cytokines in inflammation," Immunity, vol. 28, no. 4, pp. 454-467, 2008.

[25] E. Dima, O. Koltsida, P. Katsaounou et al., "Implication of Interleukin (IL)-18 in the pathogenesis of chronic obstructive pulmonary disease (COPD)," Cytokine, vol. 74, no. 2, pp. 313-317, 2015. 


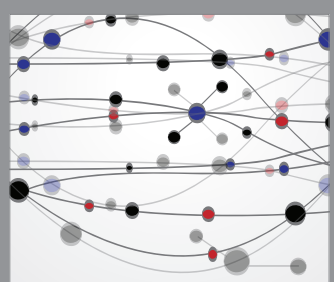

The Scientific World Journal
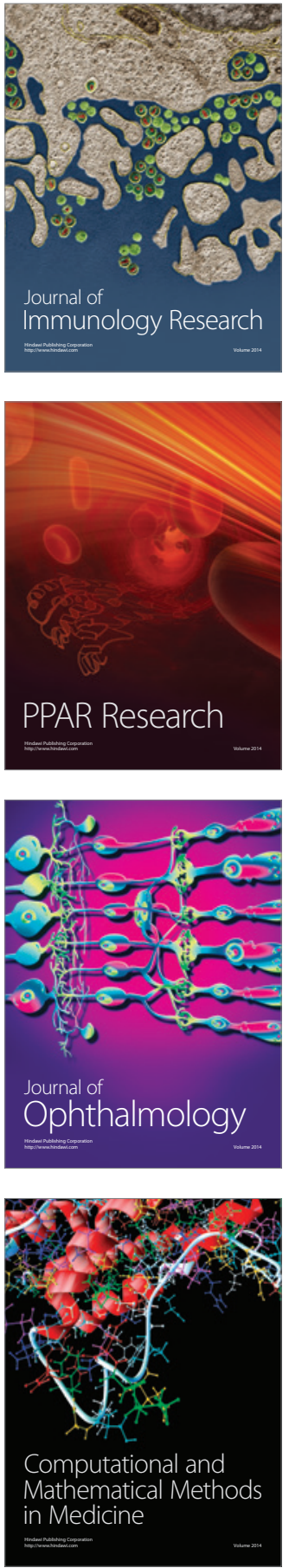

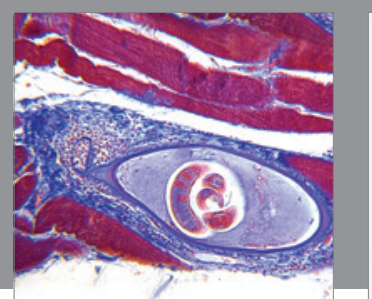

Gastroenterology Research and Practice

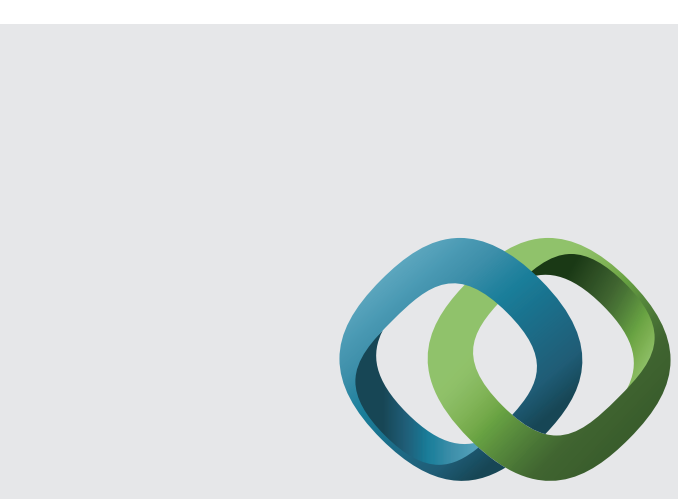

\section{Hindawi}

Submit your manuscripts at

http://www.hindawi.com
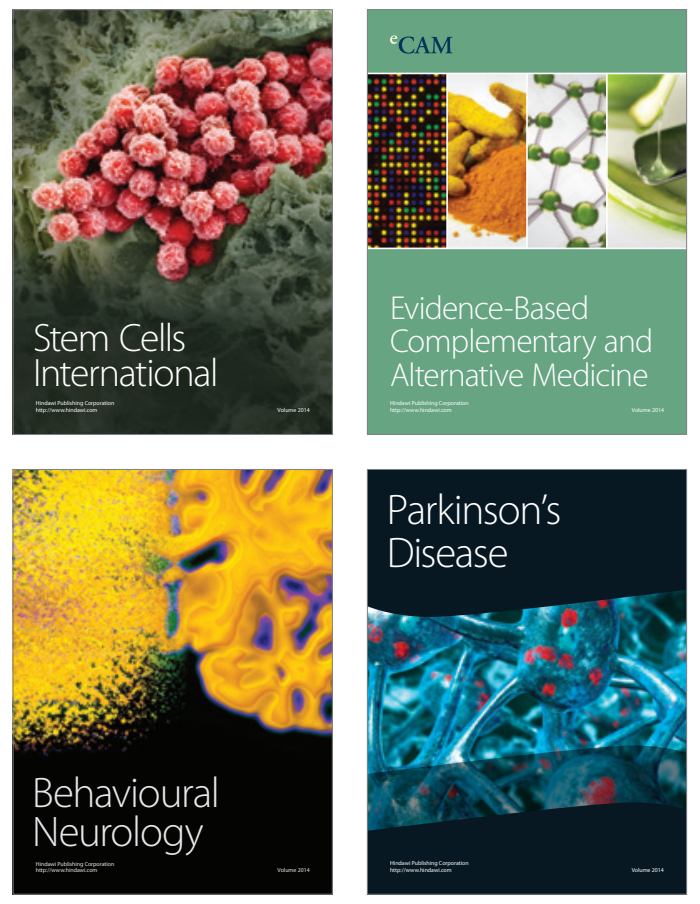
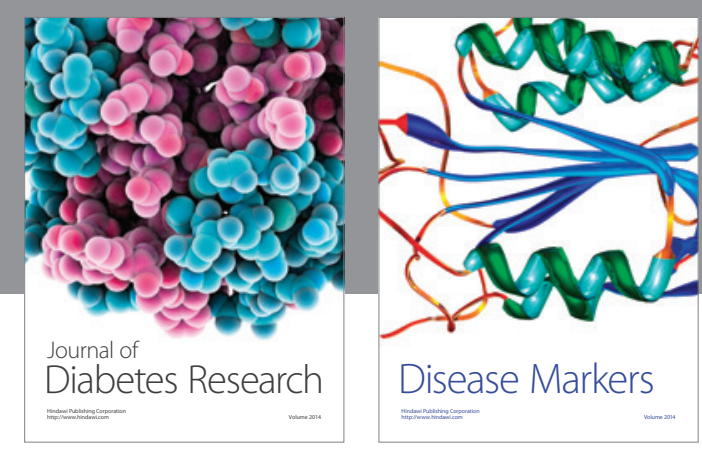

Disease Markers
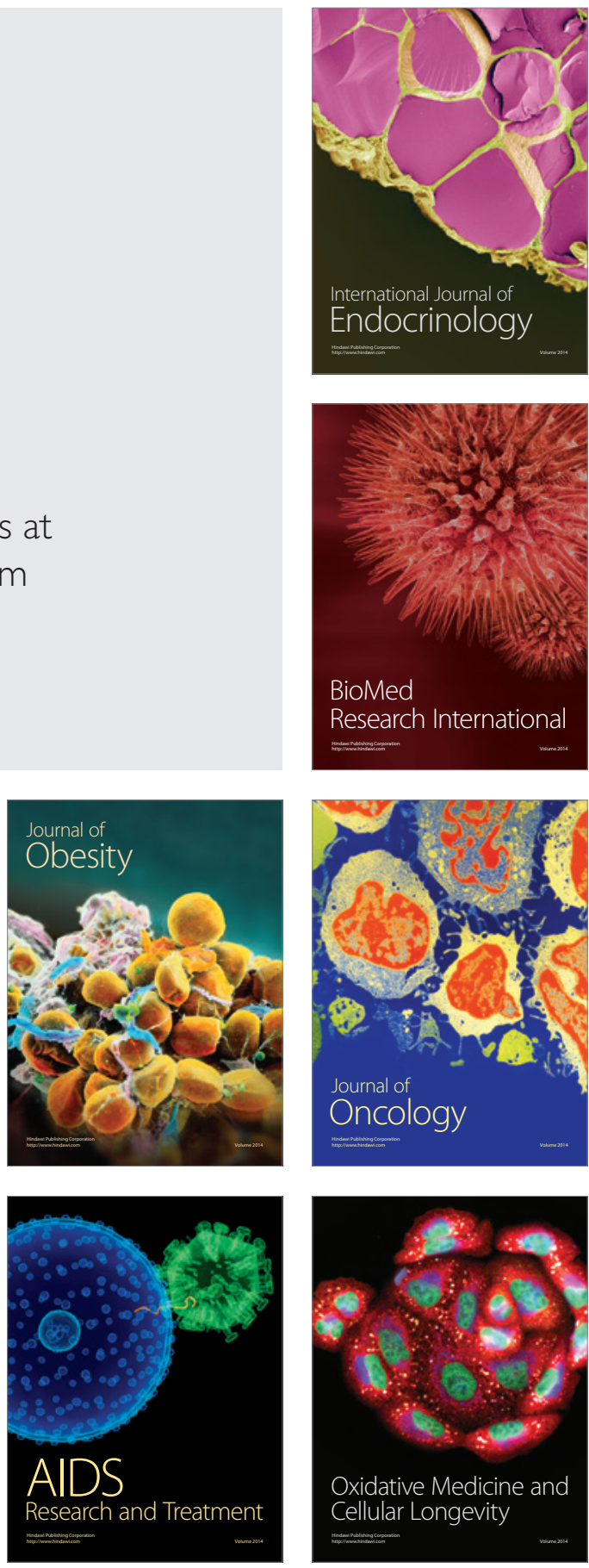